\title{
Maternal Anxiety and Depression during Late Pregnancy and Newborn Brain White Matter Development
}

\author{
(D) R.M. Graham, (D). Jiang, (D) G. McCorkle, (D)B.J. Bellando, (D).T. Sorensen, (D).M. Glasier, (D) R.H. Ramakrishnaiah, (D)A.C. Rowell,
} (iD).L. Coker, and (D)X. Ou

\begin{abstract}
BACKGROUND AND PURPOSE: Anxiety and depression during pregnancy have been associated with an increased risk of adverse neurodevelopmental outcomes in offspring. We aimed to study the in utero effects of maternal anxiety and depression on early brain development.

MATERIALS AND METHODS: Pregnant women were recruited at $\sim 36$ weeks of gestation for this prospective study. They were assessed for anxiety symptoms by the State-Trait Anxiety Inventory and for depression symptoms by the Beck Depression Inventory, 2nd Edition. After delivery, infant underwent an MR imaging examination of the brain without sedation, including DTI, for evaluation of white matter (WM) development. Infant fractional anisotropy values, a putative marker of WM integrity, were correlated with the mothers' State-Trait Anxiety Inventory and Beck Depression Inventory scores by using both tract-based spatial statistics and ROI methods.

RESULTS: Thirty-four infants were included in this study. Both maternal State-Anxiety and Trait-Anxiety scores negatively correlated $(P<.05$, corrected) with fractional anisotropy values in widespread brain WM regions; Beck Depression Inventory scores also negatively correlated $(P<.05)$ with fractional anisotropy values in one cluster in the brain. Further ROI analyses confirmed significant negative correlations between average fractional anisotropy values in ROls including left and right prefrontal WM, left and right middle frontal gyrus WM, and the fornix, and State-Anxiety ( $R$ values, -0.47 to $-0.67 ; P$ values, .008 to $<.001$ ), Trait-Anxiety ( $R,-$ 0.37 to $-0.59 ; P, .04$ to $<.001)$, and Beck Depression Inventory ( $R$ values, -0.36 to $-0.55 ; P, .05$ to .002$)$ scores.
\end{abstract}

CONCLUSIONS: Higher maternal anxiety and depression symptom scores during late pregnancy were associated with lower estimated infant brain WM development, which indicated in utero influences of maternal mental health during pregnancy on the developing brain.

ABBREVIATIONS: BDI-II = Beck Depression Inventory, 2nd ed; FA = fractional anisotropy; S-Anxiety = State-Anxiety score; SD = standard deviation; STAI $=$ State-Trait Anxiety Inventory; T-Anxiety $=$ Trait-Anxiety score; TBSS $=$ tract-based spatial statistics

I: is estimated that $28.8 \%$ of the US population is diagnosed with an anxiety disorder and that $20.8 \%$ will be diagnosed with a mood disorder, for example, depression, throughout life. ${ }^{1}$ These disorders are of particular concern to women of reproductive age, which coincides with the median age of onset for these diagnoses, ${ }^{1}$ because the physiologic and metabolic

Received April 7, 2020; accepted after revision July 6.

From the Departments of Radiology (R.M.G., L.J., C.M.G., R.H.R., A.C.R., X.O.) Pediatrics (B.J.B., S.T.S., C.M.G., R.H.R., X.O.), and Psychiatry, and Obstetrics and Gynecology (J.L.C.), University of Arkansas for Medical Sciences, Little Rock, Arkansas; Arkansas Children's Nutrition Center (G.M., X.O.), Little Rock, Arkansas; and Arkansas Children's Research Institute (X.O.), Little Rock, Arkansas.

This study was supported in part by National Institutes of Health (NIH) IP20GM121293-6283, NIH R01HD099099, and U.S. Department of Agriculture/ Agricultural Research Service 6251-51000-006-00D.

Please address correspondence to Xiawei Ou, PhD, Department of Radiology, University of Arkansas for Medical Sciences, 1 Children's Way, Slot 105, Little Rock, AR 72202; e-mail: ouxiawei@uams.edu

- Indicates open access to non-subscribers at www.ajnr.org

http://dx.doi.org/10.3174/ajnr.A6759 changes associated with pregnancy may play an important role in triggering these conditions. In fact, it has been estimated that $\sim 13 \%$ of pregnant women in the United States experience an anxiety-related disorder, and $13.3 \%$ have a mood disorder, for example, depression. ${ }^{2,3}$

Maternal anxiety and depression during pregnancy are not only a significant health issue for the women because many of them continue to have psychiatric disorders postpartum, but also a potential health concern for their offspring. For example, offspring of women who have depression during pregnancy are almost 5 times more likely to receive a diagnosis of depression by age 16 than those who were not exposed to antenatal depression. ${ }^{4}$ In addition, increased maternal anxiety during early pregnancy is independently associated with lower mental development scores of infants at age 1 year ${ }^{5}$ as well as hyperactivity and inattention in boys at 4 years of age, and behavioral and emotional problems in both boys and girls. ${ }^{6}$ 
Recent advances in noninvasive neuroimaging, particularly in quantitative MR imaging methods, ${ }^{7}$ provide an opportunity to link maternal depression and anxiety with adverse neurodevelopmental outcomes in offspring by sensitive evaluation of children's early brain development. A few new studies have revealed associations between prenatal anxiety and/or depression and infant brain structural or functional development measured by MR imaging, ${ }^{8-10}$ which suggests the possibility of in utero influences of a mother's mental health status to fetal brain development. In addition, maternal anxiety and depression are associated with an increased risk of fetal growth restriction, ${ }^{11}$ which has been linked to changes in later brain development. ${ }^{12}$

These possible in utero influences reflected by brain differences at early ages may be key to understand the relationships between prenatal maternal mental health and long-term neurodevelopment outcomes because studies have indicated the predictive value of early brain measures to later outcomes. ${ }^{13,14}$ Moreover, studies also reported associations between maternal anxiety and/or depression during pregnancy and their offspring's brain structure and function at later ages, ${ }^{15,16}$ although other postnatal influences may have confounded these relationships. Overall, with emerging evidence that shows the potential impact of maternal anxiety and depression on offspring neurodevelopment, more studies are needed to characterize how different brain regions may be vulnerable to this unfavorable prenatal environment and to delineate the prenatal effects with postnatal influences.

In this study, we hypothesized that both maternal anxiety and depression during pregnancy would negatively affect offspring fetal brain development, particularly for brain regions that control emotion, motivation, memory, and cognitive and/or executive functions. We aimed to determine if significant associations exist between measures of symptoms for these disorders in late pregnancy and measures of infant WM development soon after birth. To achieve that, we recruited healthy pregnant women without complications, measured their anxiety and depression symptomatology by using standardized tests, and evaluated WM microstructural development in their newborn infants during the first few weeks of life by using diffusion-tensor MR imaging.

\section{MATERIALS AND METHODS \\ Subjects}

All study procedures were approved by the institutional review board of the University of Arkansas for Medical Sciences, and all the participants signed informed consent to be included in this study. Healthy pregnant women without medical complications during pregnancy were recruited through the Arkansas Children's Nutrition Center for this prospective study. The inclusion criteria for the pregnant women were the following: singleton pregnancy, $\sim 36$ weeks of pregnancy, and $\geq 18$ years of age. Exclusion criteria were the following: hypertension, diabetes, or other pre-existing medical conditions known to influence fetal growth; self-reported recreational drug, tobacco, or alcohol use while pregnant; pregnancy conception with assisted fertility treatment; and medical conditions developed during pregnancy known to influence fetal growth. Infants born preterm, with congenital defects and/or anomalies (or known chromosomal abnormalities), small for gestational age (birth weight $<10$ th percentile), with a
Demographic information of the infants (17 male and 17 female) and their mothers' STAI, BDI-II, and IQ test scores at $\sim 36$ weeks of pregnancy

\begin{tabular}{lcc}
\hline & \multicolumn{2}{c}{ Result } \\
\cline { 2 - 3 } \multicolumn{1}{c}{ Demographic } & Mean \pm SD & $\begin{array}{c}\text { Range: minimum, } \\
\text { maximum }\end{array}$ \\
\hline Gestational age at birth, days & $275 \pm 7$ & 259,285 \\
Age at MR imaging, days & $18 \pm 7$ & 12,37 \\
Mother's IQ score & $99 \pm 11$ & 74,132 \\
Mother's S-Anxiety score & $29 \pm 8$ & 20,52 \\
Mother's T-Anxiety score & $34 \pm 10$ & 21,61 \\
Mother's BDI-II score & $9 \pm 6$ & 0,29 \\
\hline
\end{tabular}

low Apgar score $(<7)$ or any other medical complications at birth that suspected to affect brain WM development were also excluded. In total, 44 infants had an MR imaging examination at age 2 weeks. Among them, 34 had both valid structural and/or diffusion scans and maternal anxiety scores ( 2 of these did not have valid depression scores) and were included in this report. The demographics for the included subjects are presented in the Table.

\section{Anxiety and Depression Assessment}

Anxiety and depression symptom scores were obtained by using the State-Trait Anxiety Inventory (STAI) and the Beck Depression Inventory, 2nd ed (BDI-II), ${ }^{17}$ respectively. Both assessments were administered by a licensed psychological examiner (GM). The STAI provides 2 results: state (S-Anxiety) and trait (T-Anxiety). The S-Anxiety score reflects the subject's current state of anxiety, that is, how the subject is feeling at the moment of filling out the evaluation. In contrast, the T-Anxiety result reflects how prone to anxiety the subject is, which can include general states of calmness and security. ${ }^{18}$ The STAI assessment consists of 40 questions in total, with 20 dedicated to each subcategory. The result of the examination is a range of scores between 20 and 80, with a higher score indicating greater anxiety. Although the STAI manual does not indicate actual cutpoints to describe clinical thresholds, results of some studies suggested a score of 39-40 to define clinically significant symptoms of S-Anxiety, ${ }^{19}$ and results of other studies suggest a score of 45 as the cutoff for T-Anxiety. ${ }^{20}$ For the purposes of this study, mean +1 standard deviation (SD) (which provided a cutoff value of 38 for S-Anxiety and 45 for T-Anxiety, similar to the literature above) was used to determine whether their endorsement of anxiety symptoms was in the elevated range. The instrument manual reveals that alpha coefficients for the STAI show correlations of 0.92 to 0.94 , which indicate that the STAI has very good internal consistency and is a reliable instrument for measuring anxiety. Validity evidence for the STAI also shows that this measure correlates well with other widely used measures of personality and adjustment and anxiety. ${ }^{21}$

The BDI-II is a 21-item questionnaire that evaluates the presence of symptoms for depression listed in the Diagnostic and Statistical Manual of Mental Disorders, 4th ed. The assessment includes items that measure affective, vegetative, cognitive, and somatic symptoms of depression during the past 2 weeks. ${ }^{22}$ The Beck Depression Inventory is one of the most widely used indexes of depression due to its high validity, internal 
consistency, and sensitivity to change. ${ }^{23}$ Overall, the BDI-II has an internal consistency of 0.9 and a retest reliability range from 0.73 to 0.96 . Validity research for the BDI-II when comparing it with the Beck Depression Inventory and other well-researched depression inventories shows excellent validity, which justifies its use for assessment of depression in these subjects. ${ }^{24}$ Results are considered to indicate the presence of "minimal" depressive symptoms for scores 0-13, "mild" depressive symptoms for scores 14-19, "moderate" for scores 20-28, and "severe" for scores $>29$.

In addition, all pregnant women were administered the Wechsler Abbreviated Scale of Intelligence, 2 nd ed, ${ }^{25}$ which is an abbreviated scale of intellectual performance that measures verbal comprehension, perceptual reasoning, and general cognitive abilities (Full Scale IQ Index). The average reliability coefficient in adult samples when calculated with the Fisher $z$ transformation is 0.97 . Concurrent validity has been established with several widely used measures of intelligence.

\section{MR Imaging Data Acquisition}

At approximately age 2 weeks, the infants were brought to the radiology department of the Arkansas Children's Hospital for an MR imaging examination of the brain at natural sleep without sedation. Mini-muffs and/or headsets were placed over their ears to protect them from the noise during the scan. They were swaddled by using a MedVac infant immobilizer and warm sheets and were securely positioned. An MR imaging-compatible camera was used to monitor them. The infants were scanned by using a PRISMA scanner (Siemens Medical Solutions) and an equipped 20-channel head coil. Pulse sequences included sagittal T1 MPRAGE (TR, $1550 \mathrm{~ms}$; TE, $3 \mathrm{~ms}$; TI, $1100 \mathrm{~ms}$; flip angle, $15^{\circ}$; voxel size, $1 \mathrm{~mm} \times 1 \mathrm{~mm} \times$ $1 \mathrm{~mm}$ ) and T2 TSE (TR, 10,500 ms; TE, $168 \mathrm{~ms}$; echo spacing, $15.3 \mathrm{~ms}$; voxel size, $1 \mathrm{~mm} \times 1 \mathrm{~mm} \times 2 \mathrm{~mm}$ ) to screen for structural abnormalities by neuroradiologists, and DWI with TR of $10,200 \mathrm{~ms}$, TE of $63 \mathrm{~ms}$, acquisition voxel size of $2 \mathrm{~mm} \times 2 \mathrm{~mm} \times$ $2 \mathrm{~mm}$, and b-value of $1000 \mathrm{~s} / \mathrm{mm}^{2}$ with diffusion-weighting gradients uniformly distributed (ie, projection of diffusion directions uniformly distributed on a sphere unit) in 30 directions to evaluate brain WM development.

\section{MR Imaging Data Analysis}

Fractional anisotropy (FA) maps were calculated by using the scanner software and were exported to a workstation with FSL (version 6.0, http://www.fmrib.ox.ac.uk/fsl) installed on a VMware Linux virtual machine (VMware) for postprocessing. The postprocessing methods were similar to those in previous publications. ${ }^{13,26,27}$ Briefly, through the use of FSL's tract-based spatial statistics (TBSS; https://fsl.fmrib.ox.ac.uk/fsl/fslwiki/TBSS) toolbox, each FA image set was eroded slightly to remove the thin and bright voxels that surround the edge of the brain, and end slices were zeroed to remove outliers, which are voxels outside the brain with noisy FA. The FA image sets were then registered to each other by using nonlinear transformation to find the most representative one, which then, consequently, served as the target images. Each FA image set was then centered and layered on top of the target images, and a mean FA map and a mean WM skeleton (with $\mathrm{FA} \geq 0.15$ as the threshold, when considering that infants have lower FA values than adults, for which 0.2 is usually the default threshold) were then generated for all the subjects and served as age-specific templates. All FA maps were then projected onto the FA skeleton to create a $4 \mathrm{D}$ FA maps dataset that encompassed all the subjects and, subsequently, was used for statistical analysis. Finally, voxelwise correlation analyses were used in TBSS to evaluate associations between FA values and STAI and BDI-II scores. In addition, WM ROI, showing consistent correlations between FA values and STAI and BDI-II scores in the TBSS analysis were sketched on the mean FA maps based on anatomy, and the average FA values for WM tracts in each ROI were calculated. The associations between these average FA values in these ROIs and STAI and BDI-II scores were also evaluated.

\section{Statistical Analysis}

For the voxelwise correlation analyses using TBSS, randomization with 5000 permutations was used. The threshold-free cluster enhancement option was used to identify voxels with significant correlation $(P<.05$, corrected for multiple comparisons in the voxelwise analysis) between FA values and S-Anxiety, T-Anxiety, or BDI-II scores. Potential confounders were considered, including postmenstrual age (gestational age at birth plus age at MR imaging), which has shown strong effects on infant WM development ${ }^{26}$ as well as maternal IQ and infant sex. These parameters were included in the randomized design matrix, and their potential confounding effects were controlled by regressing out before permutation. For the ROI analysis, partial Spearmen correlation analyses were used to evaluate correlations between average FA values in each ROI and the S-Anxiety, T-Anxiety, and BDI-II scores, also with the effects of potential confounders (postmenstrual age at MR imaging, infant sex, mother's IQ) controlled. Both the correlation coefficients and the $P$ values were computed and presented. $P<.05$ was regarded as significant.

\section{RESULTS}

The characteristics for the participants included in this study are listed in the Table. Most of the mothers (76\%) self-reported anxiety and depressive symptoms that were within normal ranges, with overall average scores for the study group also well within established normal values. In total, 8 mothers (24\%) reported elevated symptoms of depression and/or anxiety. Specifically, among 5 women (15\%) with elevated depressive symptoms, 1 had severe symptoms and 4 had mild symptoms on the BDI-II. Anxiety was also considered clinically elevated in 5 women (15\%) on the T-Anxiety score and 1 woman $(3 \%)$ on the S-Anxiety score. There were 2 women (6\%) who endorsed clinical elevations on both anxiety and depression measures. There was 1 woman who was taking sertraline (Zoloft; a selective serotonin reuptake inhibitor) $50 \mathrm{mg}$ once a day and another woman who was taking Zoloft $25 \mathrm{mg}$ per day during pregnancy to treat anxiety. No other women took anxiety or depression treatment medicine during pregnancy. In addition, the S-Anxiety and T-Anxiety scores showed strong correlation (correlation coefficient $R=0.78, P<$ $.001)$, and both S-Anxiety $(R=0.61, P<.001)$ and T-Anxiety $(R=0.65, P<.001)$ scores showed significant correlations with Beck Depression Inventory scores. 

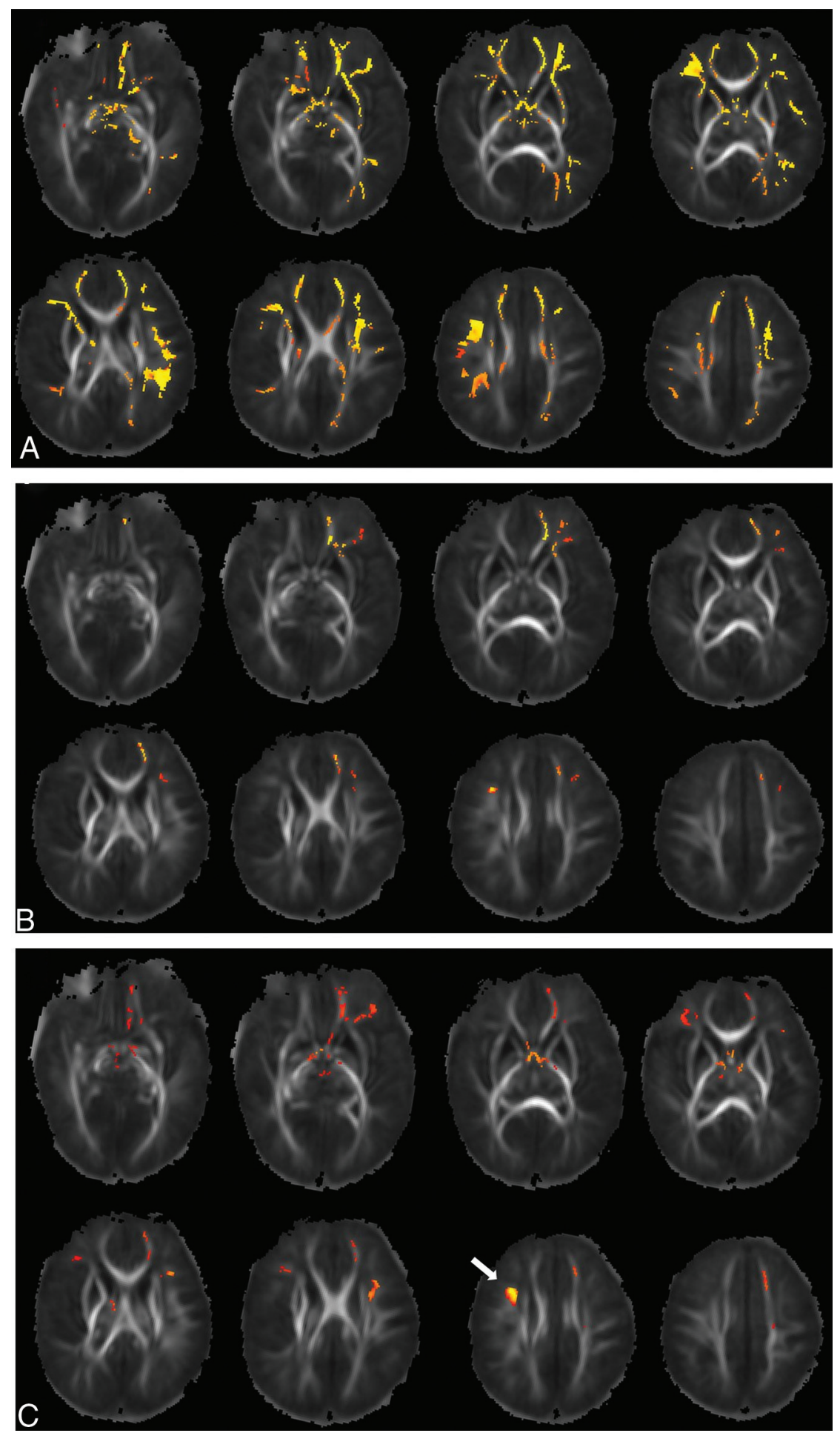

FIG 1. $A$, WM regions (orange/red) showing significant $(P<.05$, corrected) negative correlations between the mother's S-Anxiety score $(n=34)$ and infant FA value. B, WM regions showing significant negative correlations ( $P<.05$, corrected) between the mother's T-Anxiety score $(n=34)$ and infant FA value. $C$, WM regions showing a trend $(P<.10$, corrected, except for the cluster shown by the white arrow in which $P<.05)$ of negative correlations between mother's BDI-II score $(n=32)$ and infant FA value.

Seventeen male and 17 female infants, born at a mean \pm SD gestational age of $275 \pm 7$ days, had successful MR imaging, with valid structural and diffusion imaging data at a mean $\pm \mathrm{SD}$ age of $18 \pm 7$ days. There were no significant incidental findings that suggested WM abnormality for any of these subjects. Voxelwise TBSS analysis showed negative correlations $(P<.05$, corrected for the voxelwise multiple comparison and controlled for postmenstrual age at MR imaging, infant sex, and mother's IQ) between SAnxiety scores in the pregnant women and $\mathrm{FA}$ values in the infant in widespread brain WM regions (Fig 1A). These regions involved frontal, parietal, and temporal WM in both brain hemispheres as well as the limbic system. In addition, the T-Anxiety score in the pregnant women also negatively and significantly $(P<.05$, corrected for multiple comparison and controlled for covariates) correlated with FA values in their infants in multiple WM regions (Fig 1B). These regions involved the left orbitofrontal, prefrontal, and middle frontal WM, and the right middle frontal WM. There also was a negative and significant $(P$ $<.05$, corrected for multiple comparison and controlled for covariates) correlation between the BDI-II score in the pregnant women and FA values in their infants in 1 cluster located in the right middle frontal WM (arrow in Fig $1 C)$. In addition, multiple $\mathrm{WM}$ regions in the left and right frontal WM as well as in the limbic system showed trends $(P<.10$, corrected for multiple comparison and controlled for covariates) of negative correlations between BDI-II scores and FA values in the TBSS analysis (Fig 1C). No imaging voxels or clusters in the brain showed a positive correlation $(P<.05)$ between the S-Anxiety, T-Anxiety, BDI-II scores, and the FA values. ${ }^{26}$

Based on the TBSS results, several regions that consistently showed clusters with significant (or trend of) correlations between the S-Anxiety, TAnxiety, BDI-II scores, and the FA values were chosen for additional ROI analysis. These ROIs were manually drawn on the mean FA maps based on anatomy and included the left-right prefrontal WM, the left-right middle frontal gyrus WM, and the fornix in the limbic system. The average FA values for the WM tracts in each of these ROIs were calculated and correlated with STAI and BDI-II scores. Specifically, 

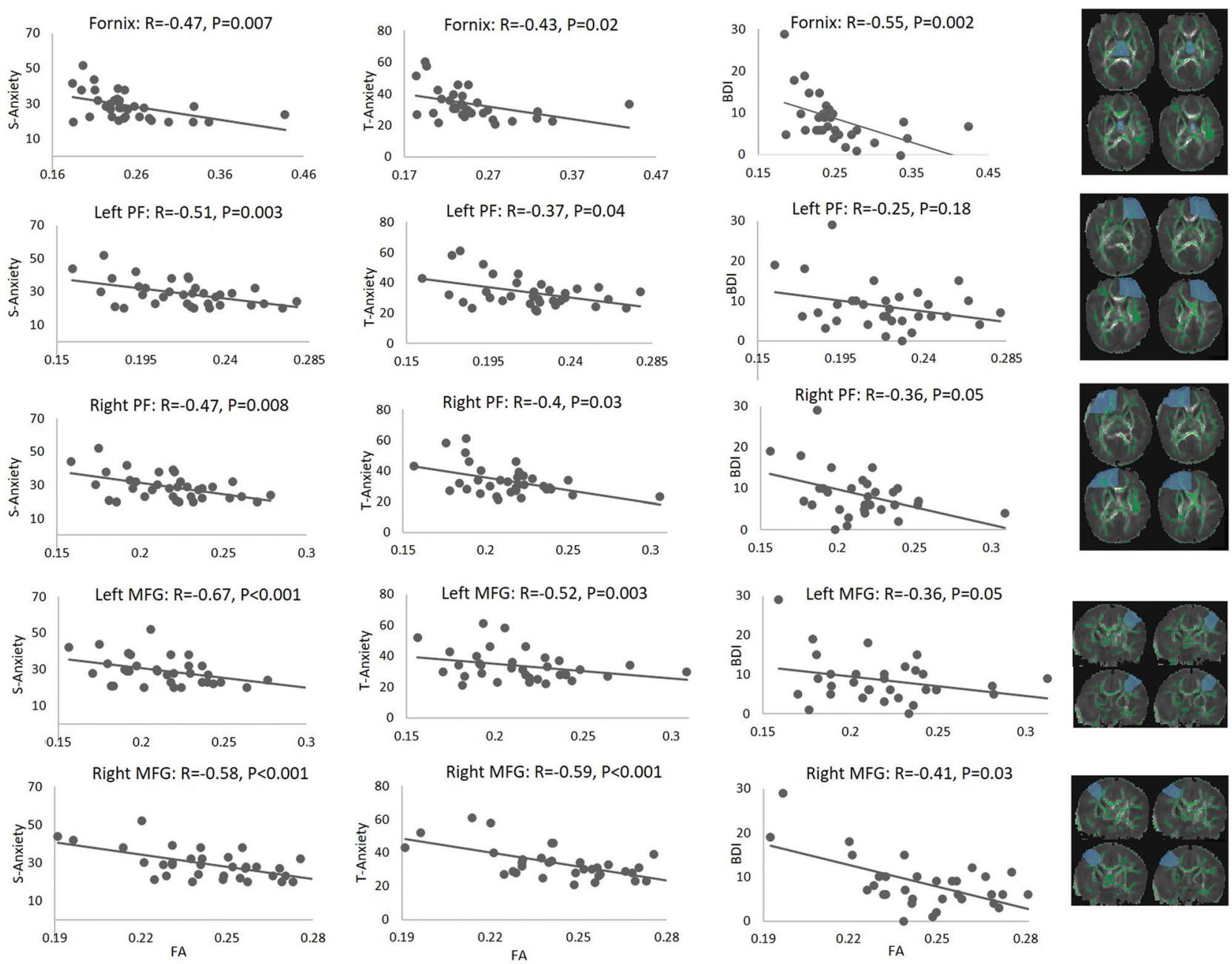

FIG 2. Correlations between infant average FA values in the selected ROls and maternal anxiety and/or depression symptom scores (S-Anxiety, T-Anxiety, BDI-II) measured at pregnancy. The column on the right shows manually drawn regions (shaded blue), which, in combination with the underlying WM skeleton (green), defined the WM ROls used for calculation. PF indicates prefrontal WM; MFG, middle frontal gyrus WM.

a partial correlation test (with the postmenstrual age at MR imaging, infant sex, and mother's IQ as covariates) showed negative correlations (correlation coefficient $\mathrm{R}$ values ranging from -0.47 to $-0.67 ; P$ values ranging from .008 to $<.001$ ) between $S$ Anxiety scores in the pregnant women and the average FA values in all of the 5 ROIs (Fig 2); negative correlations (correlation coefficient $\mathrm{R}$ values ranging from -0.37 to -0.59 ; $P$ values ranging from .04 to $<.001$ ) between $\mathrm{T}$-Anxiety scores in the pregnant women and average FA values in all of the 5 ROIs (Fig 2); and negative correlations (correlation coefficients $\mathrm{R}$ values ranged from -0.36 to -0.55 ; $P$ values ranged from .05 to .002 ) between BDI-II scores in the pregnant women and the average FA values in 4 of the 5 ROIs (Fig 2).

If depression scores were controlled for, then the relationships between the anxiety scores and the FA values weakened. Nevertheless, the following relationships were still significant: SAnxiety and left prefrontal WM FA $(R=-0.40, P=.03)$, or left $(R=-0.59, P<.001)$ or right $(\mathrm{R}=-0.42, P=.03)$ middle frontal gyrus WM FA; and T-Anxiety and left $(R=-0.43, P=.02)$ or right $(R=-0.38, P=.05)$ middle frontal gyrus WM FA. On the other hand, if anxiety scores were controlled for, then the relationships between the depression scores and the FA values weakened as well, with only the relationship between the BDI-II score and fornix FA significant $(R=-0.40, P=.04)$. Therefore, it seems that associations in the left prefrontal and left-right middle frontal gyrus WM may be more specific to anxiety, whereas the associations in the fornix may be more specific to depression.

\section{DISCUSSION}

Our prospective study showed that the healthy full-term infants born from uncomplicated pregnancies of mothers with symptoms for anxiety or depression (higher S-Anxiety, T-Anxiety, or BDI-II scores) had lower estimated WM development (as indicated by lower FA values) in multiple brain regions. A number of recent brain imaging studies looked into the relationships between maternal anxiety or depression during pregnancy and measurements of brain structure or function in children. For example, maternal anxiety during the second trimester of 
pregnancy was associated with decreased GM volume in the prefrontal cortex and several other cerebral-cerebellar regions in 69-year-old children. ${ }^{15}$ Likewise, depression symptoms at the second trimester measured by the Edinburgh Postnatal Depression Scale negatively correlated with cortical thickness in inferior frontal and middle temporal regions in 2.6-5.1-year-old children. ${ }^{16}$ In addition, increased maternal anxiety measured at 26 weeks of pregnancy using the STAI was associated with slower growth of the hippocampus in the infant brain during the first 6 months of life. ${ }^{28}$ Functionally, maternal depressive symptoms measured by the Edinburgh Postnatal Depression Scale at 26 weeks of gestation were also associated with lower functional connectivity between the amygdala and the corticostriatal circuitry in girls at 4 years of age. ${ }^{29}$ Importantly, although postpartum maternal depression and a few other postnatal factors were considered in the aforementioned studies, the associations between in utero exposure to maternal depression and anxiety and brain development in childhood were inevitably confounded by many other postnatal factors, such as family environment and child nutrition. Measuring the neonatal brain soon after birth may be more indicative of the in utero effects, independent of postnatal influences. In fact, a few imaging studies with slightly different designs when compared with our study (eg, time evaluating pregnant women and infants, structural and/or microstructural parameters analyzed) looked into whether brain structures in young infants show changes associated with maternal depression or anxiety during pregnancy. For example, FA values in the amygdala in 614-day-old infants were lower for those born to mothers with high depression symptom scores during pregnancy versus those born to mothers with low depression symptom scores. ${ }^{9}$ Similarly, a diffusion imaging study on 1-month-old infants showed that a higher composite symptom score of anxiety and/or depression measured at the third trimester of pregnancy in their mothers was associated with lower microstructural measures in their frontal WM. ${ }^{8}$ Interestingly, although widespread differences in WM microstructures in 1-month-old infants were found between those born to mothers who received depression treatment with selective serotonin reuptake inhibitors during pregnancy compared with controls (mothers with no depression diagnosis or treatment during pregnancy), there were no differences in WM microstructures or GM volumes when comparing those born to mothers with a history of depression but no treatment with the same controls. ${ }^{30}$ Analysis of these results suggests that exposure to medicine may have important confounding effects in relationships of maternal anxiety and/or depression and fetal brain development, and should not be overlooked. Analysis of these results also suggested that additional studies may be necessary to confirm the in utero effects of anxiety and depression on brain development.

Our voxel wise TBSS and subsequent ROI analysis results indicated a few brain regions that consistently showed negative correlations between higher anxiety and/or depression symptoms and lower estimated WM development. These regions mainly involved the left and right prefrontal WM, the left and right middle frontal WM, and the fornix in the limbic system. The findings in prefrontal-middle frontal WM are consistent with a few previous studies. ${ }^{8,15,16}$ The prefrontal cortex is important for executive functioning, attention, and memory as well as speech production and language. Depression and/or anxiety has been linked with abnormalities in prefrontal $\mathrm{WM},{ }^{31-33}$ and exposure to maternal depression and/or anxiety in utero has been linked to adverse development in executive functioning. ${ }^{34}$ Likewise, WM abnormalities associated with anxiety and/or depression have been found in the middle frontal gyrus, ${ }^{32}$ which supposedly serves as the connection between the ventral and dorsal attention network and acts as a "circuit-breaker" to transition between stimulus-driven and goal-oriented attention; ${ }^{35}$ and, studies show that antenatal maternal depression and/or anxiety were associated with a higher risk of child attention problems. ${ }^{36}$ The associations between maternal anxiety and/or depression during pregnancy and offspring fornix development have not been reported before, with only one study showing changes of pace in hippocampal volume growth in infants associated with maternal anxiety. ${ }^{28}$ Development of the fornix begins at week 10 of gestation and continues rapidly throughout gestation as one of the most prominent brain fiber tracts at the fetal stage despite fornix's relatively small size in the adult brain. ${ }^{37}$ It is understandable that the fornix is vulnerable to environmental influences during this stage of rapid development. In addition, lower fornix FA values at birth have been associated with lower cognitive scores measured at 2 years of age, ${ }^{13}$ which indicate the functional importance of normal early development of this structure.

Although the underlying mechanism for the changes in in utero WM development associated with maternal anxiety or depression is unclear, there are a few physiologic and/or metabolic factors that may be involved. The increase of cortisol (one of the major stress and anxiety hormones) level in the maternal and fetal circulation may affect fetal neural development. ${ }^{38}$ In addition, maternal anxiety and/or depression may lead to an increased number of inflammatory cytokines in circulation, which also could adversely affect fetal development via alteration of the in utero environment. ${ }^{39}$ Although these potential pathways can only be speculated without appropriately designed studies, our imaging data that show infant brain differences associated with maternal anxiety and/or depression during pregnancy indicate that there are likely fetal programming effects involved. One clinical implication of our study is that focus on postpartum mental health of women at reproductive age may need to be extended to before and during pregnancy because there may be significant prenatal influences on offspring brain development.

There are a few limitations with our study. First, the sample size was small. Although significant associations between maternal anxiety and/or depression and infant WM development were revealed by using this small cohort, a larger cohort can allow consideration to more potential confounders and may potentially identify more regions with significant correlation. Second, the psychological evaluations of anxiety and depression symptoms were only measured once, at 36 weeks of pregnancy. Future studies with longitudinal observation of these symptoms throughout the pregnancy may provide a more complete characterization of fetal exposure to this unfavorable environment. Third, in our study design, we included participants on medicine for treatment of anxiety or depression $(n=2)$. Although the effects of medicine did not seem to change our results (when analyzing the data by 
adding whether taking medicine as an additional covariate), the effects may need to be carefully considered with a larger cohort. Fourth, we only focused our diffusion evaluation on FA values that are commonly available on clinical scanners, while FA values are sensitive but not specific to different microstructural changes in WM. Nevertheless, our study is one of the first to reveal significant effects of both maternal anxiety and depression during pregnancy on offspring brain development, and in imaging the infant brain as early as possible to capture the in utero effects while limiting postnatal influences.

\section{CONCLUSIONS}

Higher maternal anxiety and depression symptom scores during late pregnancy were each associated with lower estimated infant brain WM development in multiple brain regions, including prefrontal and middle frontal WM and the fornix, indicating in utero influences and early brain changes, which may potentially lead to changes in long-term neurodevelopmental outcomes in offspring.

Disclosures: Ginger McCorkle-RELATED: Grant: NIH, Comments: This project is funded by NIH 1P20GM121293-6283.* Betty Jayne Bellando-RELATED: Grant: NIH IP20GM121293-6283.* Charles M. Glasier-UNRELATED: Consultancy: Medicolegal consulting. Amy C. Rowell-RELATED: Grant: NIH 1P20GM1212936283.* Jessica L. Coker-UNRELATED: Grants/Grants pending: Brain and Behavior Research Foundation NIH/National Institute on Drug Abuse.* Xiawei Ou-RELATED: Grant: NIH, Comments: IP20GM121293-6283, IR01HD099099.* *Money paid to the institution.

\section{REFERENCES}

1. Kessler RC, Berglund $\mathrm{P}$, Demler $\mathrm{O}$, et al. Lifetime prevalence and age-of-onset distributions' of DSM-IV disorders in the national comorbidity survey replication. Arch Gen Psychiatry 2005;62:593602 CrossRef Medline

2. Vesga-López O, Blanco C, Keyes K, et al. Psychiatric disorders in pregnant and postpartum women in the United States. Arch Gen Psychiatry 2008;65:805-15 CrossRef Medline

3. American College of Obstetricians and Gynecologists Committee on Health Care for Underserved Women. ACOG Committee Opinion No. 343: psychosocial risk factors: perinatal screening and intervention. Obstet Gynecol 2006;108:469-77 CrossRef Medline

4. Pawlby S, Hay DF, Sharp D, et al. Antenatal depression predicts depression in adolescent offspring: prospective longitudinal community-based study. I Affect Disord 2009;113:236-43 CrossRef Medline

5. Davis EP, Sandman CA. The timing of prenatal exposure to maternal cortisol and psychosocial stress is associated with human infant cognitive development. Child Dev 2010;81:131-48 CrossRef Medline

6. O'Connor TG, Heron J, Golding J, et al. Maternal antenatal anxiety and children's behavioural/emotional problems at 4 years - report from the Avon Longitudinal Study of Parents and Children. $\mathrm{Br} \mathrm{J}$ Psychiatry 2002;180:502-08 CrossRef Medline

7. Dubois J, Alison M, Counsell SJ, et al. MRI of the neonatal brain: a review of methodological challenges and neuroscientific advances. J Magn Reson Imaging 2020 May 18. [Epub ahead of print] CrossRef Medline

8. Dean DC III, Planalp EM, Wooten W, et al. Association of prenatal maternal depression and anxiety symptoms with infant white matter microstructure. JAMA Pediatr 2018;172:97381CrossRef Medline

9. Rifkin-Graboi A, Bai J, Chen H, et al. Prenatal maternal depression associates with microstructure of right amygdala in neonates at birth. Biol Psychiatry 2013;74:837-44 CrossRef Medline
10. Rifkin-Graboi A, Meaney MJ, Chen H, et al. Antenatal maternal anxiety predicts variations in neural structures implicated in anxiety disorders in newborns. I Am Acad Child Adolesc Psychiatry 2015;54:313-21.e2 CrossRef Medline

11. Grote NK, Bridge JA, Gavin AR, et al. A meta-analysis of depression during pregnancy and the risk of preterm birth, low birth weight, and intrauterine growth restriction. Arch Gen Psychiatry 2010;67:1012-24 CrossRef Medline

12. Miller SL, Huppi PS, Mallard C. The consequences of fetal growth restriction on brain structure and neurodevelopmental outcome. $J$ Physiol 2016;594:807-23 CrossRef Medline

13. Feng KY, Rowell AC, Andres A, et al. Diffusion tensor MRI of white matter of healthy full-term newborns: relationship to neurodevelopmental outcomes. Radiology 2019;292:179-87 CrossRef Medline

14. $\mathrm{Hu} \mathrm{HCH}, \mathrm{McAllister} \mathrm{AS}$. The potential and promise of diffusion tensor MRI in predicting neurodevelopment in children. Radiology 2019;292:188-89 CrossRef Medline

15. Buss C, Davis EP, Muftuler LT, et al. High pregnancy anxiety during mid-gestation is associated with decreased gray matter density in 6-9-year-old children. Psychoneuroendocrinology 2010;35:141-53 CrossRef Medline

16. Lebel C, Walton M, Letourneau N, et al. Prepartum and postpartum maternal depressive symptoms are related to children's brain structure in preschool. Biol Psychiatry 2016;80:859-68 CrossRef Medline

17. Beck AT, Steer RA, Brown GK. Manual for the Beck Depression Inventory-II. San Antonio: Psychological Corporation; 1996

18. Julian LJ. Measures of anxiety: State-Trait Anxiety Inventory (STAI), Beck Anxiety Inventory (BAI), and Hospital Anxiety and Depression Scale - Anxiety (HADS-A). Arthritis Care Res 2011;63 (suppl 11):S467-72 CrossRef Medline

19. Knight RG, Waal-Manning HJ, Spears GF. Some norms and reliability data for the State-Trait Anxiety Inventory and the Zung SelfRating Depression scale. Br J Clin Psychol 1983;22(pt 4):245-49 CrossRef Medline

20. Bunevicius A, Staniute M, Brozaitiene J, et al. Screening for anxiety disorders in patients with coronary artery disease. Health Qual Life Outcomes 2013;11:37 CrossRef Medline

21. Guillén-Riquelme A, Buela-Casal G. Meta-analysis of group comparison and meta-analysis of reliability generalization of the StateTrait Anxiety Inventory Questionnaire (STAI). Rev Esp Salud Publica 2014;88:101-12 CrossRef Medline

22. Smarr KL, Keefer AL. Measures of depression and depressive symptoms: Beck Depression Inventory-II (BDI-II), Center for Epidemiologic Studies Depression Scale (CES-D), Geriatric Depression Scale (GDS), Hospital Anxiety and Depression Scale (HADS), and Patient Health Questionnaire-9 (PHQ-9. ). Arthritis Care Res (Hoboken) 2011;63(suppl 11):S454-66 CrossRef Medline

23. Richter P, Werner J, Heerlein A, et al. On the validity of the Beck Depression Inventory - a review. Psychopathology 1998;31:160-68 CrossRef Medline

24. Wang YP, Gorenstein C. Psychometric properties of the Beck Depression Inventory-II: a comprehensive review. Braz J Psychiatry 2013;35:416-31 CrossRef Medline

25. Wechsler D. Wechsler Abbreviated Scale of Intelligence. 2nd ed. San Antonio: NCS Pearson; 2011

26. Ou X, Glasier CM, Ramakrishnaiah $\mathrm{RH}$, et al. Gestational age at birth and brain white matter development in term-born infants and children. AJNR Am J Neuroradiol 2017;38:2373-79 CrossRef Medline

27. Ou X, Thakali KM, Shankar K, et al. Maternal adiposity negatively influences infant brain white matter development. Obesity (Silver Spring) 2015;23:1047-54 CrossRef Medline

28. Qiu A, Rifkin-Graboi A, Chen H, et al. Maternal anxiety and infants' hippocampal development: timing matters. Transl Psychiatry 2013;3:e306 CrossRef Medline 
29. Soe NN, Wen DJ, Poh JS, et al. Perinatal maternal depressive symptoms alter amygdala functional connectivity in girls. Hum Brain Mapp 2018;39:680-90 CrossRef Medline

30. Jha SC, Meltzer-Brody S, Steiner RJ, et al. Antenatal depression, treatment with selective serotonin reuptake inhibitors, and neonatal brain structure: a propensity-matched cohort study. Psychiatry Res Neuroimaging 2016;253:43-53 CrossRef Medline

31. Li LJ, Ma N, Li ZX, et al. Prefrontal white matter abnormalities in young adult with major depressive disorder: a diffusion tensor imaging study. Brain Res 2007;1168:124-28 CrossRef Medline

32. Bae JN, MacFall JR, Krishnan KRR, et al. Dorsolateral prefrontal cortex and anterior cingulate cortex white matter alterations in late-life depression. Biol Psychiatry 2006;60:1356-63 CrossRef Medline

33. Kim MJ, Whalen PJ. The structural integrity of an amygdala-prefrontal pathway predicts trait anxiety. J Neurosci 2009;29:11614-18 CrossRef Medline

34. Buss C, Davis EP, Hobel CJ, et al. Maternal pregnancy-specific anxiety is associated with child executive function at 6-9 years age. Stress 2011;14:665-76 CrossRef Medline
35. Corbetta M, Shulman GL. Control of goal-directed and stimulusdriven attention in the brain. Nat Rev Neurosci 2002;3:201-15 CrossRef Medline

36. Van Batenburg-Eddes T, Brion MJ, Henrichs J, et al. Parental depressive and anxiety symptoms during pregnancy and attention problems in children: a cross-cohort consistency study. J Child Psychol Psychiatry 2013;54:591-600 CrossRef Medline

37. Huang H, Xue R, Zhang JY, et al. Anatomical characterization of human fetal brain development with diffusion tensor magnetic resonance imaging. J Neurosci 2009;29:4263-73 CrossRef Medline

38. Van den Bergh BRH, Mulder EJH, Mennes M, et al. Antenatal maternal anxiety and stress and the neurobehavioural development of the fetus and child: links and possible mechanisms. A review. Neurosci Biobehav Rev 2005;29:237-58 CrossRef Medline

39. Karlsson L, Nousiainen N, Scheinin NM, et al. Cytokine profile and maternal depression and anxiety symptoms in mid-pregnancy-the FinnBrain Birth Cohort Study. Arch Womens Ment Health 2017;20:39-48 CrossRef Medline 